\title{
ASYMPTOTIC STABILIZATION OF EULER-POINCARÉ MECHANICAL SYSTEMS
}

\author{
Anthony M. Bloch ${ }^{*, 1}$ Dong Eui Chang***,3 \\ Naomi E. Leonard ${ }^{* *, 2}$ Jerrold E. Marsden ${ }^{* * *, 3}$ \\ Craig Woolsey ${ }^{* *, 2}$ \\ * Department of Mathematics, University of Michigan, \\ Ann Arbor, MI 48109 \\ ** Department of Mechanical and Aerospace Engineering, \\ Princeton University, Princeton, NJ 08544 \\ *** Control and Dynamical Systems, California Institute of \\ Technology 107-81, Pasadena, CA 91125
}

\begin{abstract}
Stabilization of mechanical control systems by the method of controlled Lagrangians and matching is used to analyze asymptotic stabilization of systems whose underlying dynamics are governed by the Euler-Poincaré equations. In particular, we analyze asymptotic stabilization of a satellite. Copyright (c) 2000 IFAC
\end{abstract}

Keywords: Feedback stabilization, Lyapunov methods, Nonlinear control, Dissipation

\section{INTRODUCTION}

This paper develops a constructive approach to the determination of asymptotically stabilizing control laws for a class of Lagrangian mechanical systems with symmetry - systems described by the Euler-Poincaré equations. This work complements and extends the class of systems discussed in Bloch, Leonard and Marsden [1997, 1998, 1999a,b, 2000a]. Here we concentrate on the details of asymptotic stability. The paper is complementary to Bloch, Leonard and Marsden [2000b] and uses some of the ideas from Bloch, Chang, Leonard and Marsden [2000] and Woolsey and Leonard [1999].

The specific case we consider is that in which the configuration space is $Q=H \times G$, where $H$ is a

\footnotetext{
1 Research partially supported by NSF grant DMS9803181, AFOSR grant F49620-96-1-0100, and an NSF group infrastructure grant at the University of Michigan 2 Research partially supported by NSF grant BES9502477 and ONR grant N00014-98-1-0649

3 Research partially supported by AFOSR grant F4962095-1-0419
}

Lie group and $G$ is an Abelian Lie group. We also assume that the Lagrangian $L: T Q \rightarrow \mathbb{R}$ is left invariant under both $G$ and $H$, so the $G$ variables are cyclic and the controls act only on these cyclic variables.

As in our previous analysis, the guiding principle behind our methodology is to begin by considering a class of control laws that yield closed-loop dynamics which remain in Lagrangian form. The goal with this first step is to achieve stabilization within the class of conservative systems. Secondly, we append controls that are dissipative in nature to turn the conservative stabilization into asymptotic stabilization.

\section{EULER-POINCARÉ MATCHING AND STABILIZATION.}

In this section we recall the Euler-Poincaré matching theorem. This will be illustrated in $\S 4$ by the spacecraft with rotors.

The spacecraft example has two symmetry groups associated with it, as do many other examples. 
One group, which in this case is the nonabelian group $\mathrm{SO}(3)$, is associated with the rotational symmetry of the overall problem and another group, an Abelian group, is the product of several copies of $S^{1}$ associated to the rotors, which are also the control directions. In this section, we use this setting to get a concrete and readily implementable Euler-Poincaré matching theorem.

\subsection{Euler-Poincaré Matching.}

Let $L: T(H \times G) \rightarrow \mathbb{R}$ denote a given left invariant Lagrangian and $l: \mathfrak{h} \times T G \rightarrow \mathbb{R}$ be the restriction of $L$ to the Lie algebra of $H$. For a curve $h(t) \in H$ let $\eta(t)=T_{h(t)} L_{h(t)^{-1}} \dot{h}$, which we also write as $\eta(t)=h(t)^{-1} \dot{h}(t)$. We consider Lagrangians that are purely kinetic energy Lagrangians, and correspondingly, the (reduced) Lagrangian takes the form

$$
l\left(\eta^{\alpha}, \dot{\theta}^{a}\right)=\frac{1}{2} g_{\alpha \beta} \eta^{\alpha} \eta^{\beta}+g_{\alpha a} \eta^{\alpha} \dot{\theta}^{a}+\frac{1}{2} g_{a b} \dot{\theta}^{a} \dot{\theta}^{b} .
$$

Here $\eta^{\alpha}$ are the variables in $\mathfrak{h}$ and $\theta^{a}$ are the control variables. Note that $g_{\alpha \beta}, g_{\alpha a}$ and $g_{a b}$ are all constant (fixed) matrices.

The cyclic variables $\theta^{a}$ in $G$ give rise to the conserved quantity

$$
J_{a}=\frac{\partial l}{\partial \dot{\theta}^{a}}=g_{a \alpha} \eta^{\alpha}+g_{a b} \dot{\theta}^{b} .
$$

The equations of motion for the control system where the controls $u_{a}$ act in the $\theta^{a}$ directions are the controlled Euler-Poincaré equations:

$$
\begin{aligned}
\frac{d}{d t} \frac{\partial l}{\partial \eta^{\alpha}} & =c_{\alpha \delta}^{\beta} \eta^{\delta} \frac{\partial l}{\partial \eta^{\beta}} \\
\frac{d}{d t} \frac{\partial l}{\partial \dot{\theta}^{a}} & =u_{a}
\end{aligned}
$$

where $c_{\alpha \delta}^{\beta}$ are the structure constants of the Lie algebra $\mathfrak{h}$.

We choose the controlled Lagrangian to be

$$
\begin{aligned}
& l_{\tau, \sigma, \rho}=l\left(\eta^{\alpha}, \dot{\theta}^{a}+\tau_{\alpha}^{a} \eta^{\alpha}\right)+\frac{1}{2} \sigma_{a b} \tau_{\alpha}^{a} \tau_{\beta}^{b} \eta^{\alpha} \eta^{\beta} \\
& +\frac{1}{2}\left(\rho_{a b}-g_{a b}\right)\left(\dot{\theta}^{a}+g^{a c} g_{c \alpha} \eta^{\alpha}+\tau_{\alpha}^{a} \eta^{\alpha}\right) \\
& \cdot\left(\dot{\theta}^{b}+g^{b c} g_{c \beta} \eta^{\beta}+\tau_{\beta}^{b} \eta^{\beta}\right) .
\end{aligned}
$$

To preserve symmetry $\tau_{\alpha}^{a}, \sigma_{a b}$ and $\rho_{a b}$ are constant matrices.

From (2.5) we find that the controlled conserved quantity is given by

$$
\tilde{J}_{a}=\frac{\partial l_{\tau, \sigma, \rho}}{\partial \dot{\theta}^{a}}=\rho_{a b}\left(\dot{\theta}^{b}+g^{b c} g_{c \alpha} \eta^{\alpha}+\tau_{\alpha}^{b} \eta^{\alpha}\right) .
$$

The controlled Lagrangian prescribes the closedloop system, i.e., the closed-loop dynamics are the Euler-Poincaré equations corresponding to $l_{\tau, \sigma, \rho}$ :

$$
\begin{aligned}
\frac{d}{d t} \frac{\partial l_{\tau, \sigma, \rho}}{\partial \eta^{\alpha}} & =c_{\alpha \delta}^{\beta} \eta^{\delta} \frac{\partial l_{\tau, \sigma, \rho}}{\partial \eta^{\beta}} \\
\frac{d}{d t} \frac{\partial l_{\tau, \sigma, \rho}}{\partial \dot{\theta}^{a}} & =0 .
\end{aligned}
$$

To effect this closed-loop system, the control inputs $u_{a}$ must be chosen so that (2.4) and (2.8) are equivalent. Additionally, the controlled Lagrangian must satisfy matching conditions, i.e., it must be chosen so that (2.3) and (2.7) are equivalent.

We make the following assumptions:

Assumption EP-1. $\tau_{\alpha}^{a}=-\sigma^{a b} g_{b \alpha}$.

Assumption EP-2. $\sigma^{a b}+\rho^{a b}=g^{a b}$.

The following is proved in Bloch, Leonard and Marsden [2000b].

Theorem 1. Under the assumptions EP-1 and EP2 , the Euler-Poincaré equations for the controlled Lagrangian (2.7)-(2.8) coincide with the controlled Euler-Poincaré equations (2.3)-(2.4).

The control law $u_{a}$ can be determined by comparing (2.4) to the controlled conservation law

$$
\frac{d}{d t} \frac{\partial l_{\tau, \sigma, \rho}}{\partial \dot{\theta}^{a}}=\frac{d}{d t} \tilde{J}_{a}=0
$$

where $\tilde{J}_{a}$ is given by (2.6). We find

$$
u_{a}=g_{a b} \sigma^{b c} g_{c \alpha} \dot{\eta}^{\alpha} \text {. }
$$

To get a control law that is a function of velocities rather than accelerations we can substitute for accelerations from the Euler-Poincaré equations. This yields

$$
\begin{aligned}
u_{a} & =k_{a}^{\alpha} \frac{d}{d t} \frac{\partial l}{\partial \eta^{\alpha}}=k_{a}^{\alpha} c_{\alpha \delta}^{\psi} \eta^{\delta} \frac{\partial l}{\partial \eta^{\psi}} \\
& =k_{a}^{\alpha} c_{\alpha \delta}^{\psi} \eta^{\delta}\left(g_{\psi \beta} \eta^{\beta}+g_{\psi b} \dot{\theta}^{b}\right),
\end{aligned}
$$

where $k_{a}^{\alpha}$ are control gains defined by

$$
\begin{gathered}
k_{a}^{\alpha}=D_{a b} \sigma^{b c} g_{c \beta} B^{\alpha \beta}, \\
B_{\alpha \beta}=g_{\alpha \beta}-g_{\alpha b} g^{a b} g_{a \beta}, \\
D^{b a}=g^{b a}+\sigma^{b c} g_{c \beta} B^{\alpha \beta} g_{\alpha e} g^{a e} .
\end{gathered}
$$

\subsection{Euler-Poincaré Stabilization}

We now use the energy-Casimir method to determine stability (see e.g. Marsden and Ratiu [1994]). Recall that for mechanical systems, an eigenvalue analysis alone is not sufficient for determining stability.

Define $l_{0}$ on $\mathfrak{h}$ by

$$
l_{0}\left(\eta^{\alpha}\right)=\frac{1}{2} g_{\alpha \beta} \eta^{\alpha} \eta^{\beta} .
$$


A (relative) equilibrium $\eta_{e}$ for the corresponding dynamical equations satisfies the equation

$$
c_{\alpha \delta}^{\beta} \eta^{\delta} \frac{\partial l_{0}}{\partial \eta^{\beta}}=0
$$

Now suppose we have a collection of Casimir functions $C^{1}\left(M_{\alpha}\right), \cdots, C^{m}\left(M_{\alpha}\right)$ where

Now set

$$
M_{\alpha}=\frac{\partial l_{0}}{\partial \eta^{\alpha}}=g_{\alpha \beta} \eta^{\beta} \text {. }
$$

$$
E_{\Phi}=l_{0}+\Phi\left(C^{1}, \cdots C^{m}\right) .
$$

We require that the Casimir functions be chosen so that the first variation of $E_{\Phi}$ vanishes at equilibrium, i.e.,

$\left.\delta\left(E_{\Phi}\right)\right|_{\eta_{e}}=\left.g_{\alpha \beta} \delta \eta^{\beta}\left(\eta^{\alpha}+\sum_{k=1}^{m}\left(D_{k} \Phi\right) \frac{\partial C^{k}}{\partial M_{\alpha}}\right)\right|_{e}=0$.

The second variation at equilibrium is given by

$$
\begin{aligned}
\left.\delta^{2}\left(E_{\Phi}\right)\right|_{\eta_{e}} & =\left(g_{\alpha \beta}+g_{\alpha \mu} H^{\mu \nu} g_{\nu \beta}\right) \delta \eta^{\alpha} \delta \eta^{\beta}, \\
H^{\mu \nu}= & \left(\sum_{k, j=1}^{m}\left(D_{k j} \Phi\right) \frac{\partial C^{k}}{\partial M_{\mu}} \frac{\partial C^{j}}{\partial M_{\nu}}\right. \\
& \left.+\sum_{k=1}^{m}\left(D_{k} \Phi\right) \frac{\partial^{2} C^{k}}{\partial M_{\mu} \partial M_{\nu}}\right)\left.\right|_{e} .
\end{aligned}
$$

Now consider the full uncontrolled Lagrangian $l$. Using (2.3), the full system will still have $\eta_{e}$ as an equilibrium together with $\dot{\theta}_{e}^{a}$ provided

$$
c_{\alpha \delta}^{\beta} \eta_{e}^{\delta}\left(g_{\beta \delta} \eta_{e}^{\delta}+g_{\beta a} \dot{\theta}_{e}^{a}\right)=0 .
$$

This is satisfied if $c_{\alpha \delta}^{\beta} \eta_{e}^{\delta} g_{\beta a} \dot{\theta}_{e}^{a}=0$ and in particular if $\dot{\theta}_{e}^{a}=0$.

This implies, from our matching conditions, that $l_{\tau, \sigma, \rho}$ also has this equilibrium. Set

$$
\begin{aligned}
\tilde{M}_{\alpha} & =\frac{\partial l_{\tau, \sigma, \rho}}{\partial \eta^{\alpha}}=\frac{\delta l}{\delta \eta^{\alpha}}=g_{\alpha \beta} \eta^{\beta}+g_{\alpha a} \dot{\theta}^{a} \\
& =G_{\alpha \beta} \eta^{\beta}+g_{\alpha a} \rho^{a b} \tilde{J}_{b}
\end{aligned}
$$

using (2.6) and where we define

$$
G_{\alpha \beta}=g_{\alpha \beta}-g_{a \alpha} \rho^{a b} g_{b \beta} .
$$

For stability of the controlled system we use

$$
E_{\tilde{\Phi}}=l_{\tau, \sigma, \rho}+\tilde{\Phi}\left(C^{k}\left(\tilde{M}_{\alpha}\right), \tilde{J}^{a}\right)
$$

where $\tilde{\Phi}$ is a smooth function.

We next compute the first and second variations of $E_{\tilde{\Phi}}$. Using again the conserved quantities $\tilde{J}_{a}$ and assumption EP-2, we get

$$
\begin{aligned}
l_{\tau, \sigma, \rho}= & \frac{1}{2}\left(g_{\alpha \beta}+g_{a \alpha}\left(\sigma^{a b}-g^{a b}\right) g_{b \beta}\right) \eta^{\alpha} \eta^{\beta} \\
& +\frac{1}{2} \rho^{a b} \tilde{J}_{a} \tilde{J}_{b} \\
\equiv & \frac{1}{2} G_{\alpha \beta} \eta^{\alpha} \eta^{\beta}+\frac{1}{2} \rho^{a b} \tilde{J}_{a} \tilde{J}_{b} .
\end{aligned}
$$

The first variation is

$$
\begin{aligned}
\delta E_{\tilde{\Phi}}= & \left.G_{\alpha \beta} \delta \eta^{\beta}\left(\eta^{\alpha}+\sum_{k=1}^{m}\left(D_{k} \tilde{\Phi}\right) \frac{\partial C^{k}}{\partial M_{\alpha}}\right)\right|_{e} \\
& +\delta \tilde{J}_{a}\left(\rho^{a b} \tilde{J}_{b}+D_{m+a} \tilde{\Phi}\right. \\
& \left.+\sum_{k=1}^{m}\left(D_{k} \tilde{\Phi}\right) \frac{\partial C^{k}}{\partial M_{\alpha}} g_{\alpha b} \rho^{a b}\right)\left.\right|_{e}=0 .
\end{aligned}
$$

Thus, we require

$$
\begin{gathered}
\left.\left(\sum_{k=1}^{m}\left(D_{k} \tilde{\Phi}\right) \frac{\partial C^{k}}{\partial \tilde{M}_{\alpha}}\right)\right|_{e}=-\eta_{e}^{\alpha}, \quad(2.26) \\
\left.D_{m+a} \tilde{\Phi}\right|_{e}=\left.\left(-\rho^{a b} \tilde{J}_{b}+\rho^{a b} g_{\alpha b} \eta^{\alpha}\right)\right|_{e}=-\left.\dot{\theta}^{a}\right|_{e}
\end{gathered}
$$

Similarly, we can compute the second variation. Consider the case (apparently sufficient for applications) where

$\tilde{\Phi}\left(C^{1}, \cdots, C^{m}, \tilde{J}^{a}\right) \equiv \Phi\left(C^{1}, \cdots, C^{m}\right)+\Psi\left(\tilde{J}^{a}\right)$.

Accordingly, (2.27) becomes

Now define

$$
\begin{aligned}
\tilde{H}^{\alpha \beta} & =\left(\sum_{k, j=1}^{m} D_{k j} \Phi \frac{\partial C^{k}}{\partial \tilde{M}_{\alpha}} \frac{\partial C^{j}}{\partial \tilde{M}_{\beta}}\right. \\
& \left.+\sum_{k=1}^{m} D_{k} \Phi \frac{\partial^{2} C^{k}}{\partial \tilde{M}_{\alpha} \partial \tilde{M}_{\beta}}\right)\left.\right|_{e} .
\end{aligned}
$$

Then, the second variation is given by

$$
\begin{aligned}
& \left.\left.\delta^{2} E_{\tilde{\Phi}}\right|_{e} \equiv \delta^{2} E_{\Phi, \Psi}\right|_{e} \\
& =N_{\alpha \beta} \delta \eta^{\alpha} \delta \eta^{\beta}+2 P_{\alpha}^{a} \delta \eta^{\alpha} \delta \tilde{J}_{a}+R^{a b} \delta \tilde{J}_{a} \delta \tilde{J}_{b} \\
& =N_{\alpha \beta} \delta \xi^{\alpha} \delta \xi^{\beta}+\left(R^{a b}-N^{\gamma \delta} P_{\gamma}^{a} P_{\delta}^{b}\right) \delta \tilde{J}_{a} \delta \tilde{J}_{b},
\end{aligned}
$$

where

$$
\begin{aligned}
& \qquad N_{\alpha \beta}=G_{\alpha \beta}+G_{\alpha \gamma} \tilde{H}^{\gamma \delta} G_{\delta \beta}, \\
& \qquad P_{\alpha}^{a}=G_{\alpha \gamma} \tilde{H}^{\gamma \delta} g_{\delta b} \rho^{a b} \\
& R^{a b}=\rho^{a b}+\left.\left(\frac{\partial^{2} \Psi}{\partial \tilde{J}_{a} \partial \tilde{J}_{b}}\right)\right|_{e}+\tilde{H}^{\alpha \beta} g_{\alpha c} \rho^{a c} g_{\beta d} \rho^{b d}, \\
& \qquad \xi^{\alpha}=\delta \eta^{\alpha}+N^{\alpha \beta} P_{\beta}^{b} \delta \tilde{J}_{b} \text {. } \\
& \text { Definiteness of this quantity at the given equilib- } \\
& \text { rium implies nonlinear stability. Using the free- } \\
& \text { dom in choosing }\left.\left(\frac{\partial^{2} \Psi}{\partial \tilde{J}_{a} \partial \tilde{J}_{b}}\right)\right|_{e} \text { we can make the } \\
& \text { second term on the right hand side of (2.32) have } \\
& \text { whatever definiteness we require. Then, stability } \\
& \text { will be guaranteed if we can choose } \rho_{a b} \text { such that } \\
& N_{\alpha \beta} \text { is definite (under the restrictions that (2.26) } \\
& \text { and (2.27) are satisfied). }
\end{aligned}
$$


Theorem 2. Let $\eta_{e}$ be an equilibrium for the uncontrolled dynamics given by $l_{0}(2.15)$. Suppose that $\dot{\theta}_{e}$ satisfies $(2.21)$. Then, $\left(\eta_{e}, \dot{\theta}_{e}\right)$ is an equilibrium for the controlled system described by $l_{\tau, \sigma, \rho}$ (2.24). This equilibrium is Lyapunov stable for the controlled dynamics if $\rho_{a b}$ and $\Phi\left(C_{1}, \ldots, C_{m}\right)$ can be found so that (2.26) is satisfied and $G_{\alpha \beta}+$ $G_{\alpha \gamma} \tilde{H}^{\gamma \delta} G_{\delta \beta}$ is definite. Here $\tilde{\Phi}$ is assumed to be of the form (2.28).

If the equilibrium is spectrally unstable for the uncontrolled dynamics, one cannot make $g_{\alpha \beta}+$ $g_{\alpha \gamma} H^{\gamma \delta} g_{\delta \beta}$ of (2.19) definite. In the controlled setting, however, we can require that $G_{\alpha \beta}+$ $G_{\alpha \gamma} \tilde{H}^{\gamma \delta} G_{\delta \beta}$ be definite. The tensor $G_{\alpha \beta}$ is the horizontal part of the metric for the controlled system, i.e., the "controlled inertia" associated with the group $H$ variables. Since $G_{\alpha \beta}=g_{\alpha \beta}-$ $g_{a \alpha} \rho^{a b} g_{b \beta}$, it is clear how the control gain $\rho_{a b}$ enters in to provide stabilization, i.e., by modifying the inertia.

\section{DISSIPATION AND ASYMPTOTIC STABILIZATION}

To obtain asymptotic stability, we introduce an additional term in the control law to simulate dissipation as follows

$$
\begin{aligned}
\frac{d}{d t} \frac{\partial l_{\tau, \sigma, \rho}}{\partial \eta^{\alpha}} & =c_{\alpha \delta}^{\beta} \eta^{\delta} \frac{\partial l_{\tau, \sigma, \rho}}{\partial \eta^{\beta}} \\
\frac{d}{d t} \frac{\partial l_{\tau, \sigma, \rho}}{\partial \dot{\theta}^{a}} & =\dot{\tilde{J}}_{a}=u_{a}^{\text {diss }}
\end{aligned}
$$

In this case the complete control law takes the form

$$
\begin{aligned}
u_{a} & =u_{a}^{\mathrm{cons}}+D_{a b} \rho^{b c} u_{c}^{\mathrm{diss}} \\
& =u_{a}^{\mathrm{cons}}+\left(g_{a b}-k_{a}^{\alpha} g_{\alpha b}\right) \rho^{b c} u_{c}^{\mathrm{diss}}
\end{aligned}
$$

where

$$
u_{a}^{\text {cons }}=k_{a}^{\alpha} c_{\alpha \delta}^{\psi} \eta^{\delta}\left(g_{\psi \beta} \eta^{\beta}+g_{\psi b} \dot{\theta}^{b}\right)
$$

and the relationship between $\rho^{a b}$ and $k_{a}^{\alpha}$ is given by Assumption EP-2 and (2.12).

Assume that we have found Casimir functions $C^{k}\left(\tilde{M}_{\alpha}\right)$ and a function $\tilde{\Phi}\left(C^{k}, \tilde{J}_{a}\right)=\Phi\left(C^{k}\right)+$ $\Psi\left(\tilde{J}_{a}\right)$ such that the Lyapunov function

$E_{\tilde{\Phi}}=l_{\tau, \sigma, \rho}+\tilde{\Phi}\left(C^{k}, \tilde{J}_{a}\right)=l_{\tau, \sigma, \rho}+\Phi\left(C^{k}\right)+\Psi\left(\tilde{J}_{a}\right)$ yields (Lyapunov) stability of the (relative) equilibrium, $\left(\eta_{e}^{\alpha}, \dot{\theta}_{e}^{a}\right)$. Then,

$$
\begin{aligned}
\frac{d}{d t} E_{\tilde{\Phi}} & =\frac{d}{d t} l_{\tau, \sigma, \rho}+\frac{\partial \Phi}{\partial C^{k}} \dot{C}^{k}+\frac{\partial \Psi}{\partial \tilde{J}_{a}} \dot{\tilde{J}}_{a} \\
& =\left(\dot{\theta}^{a}+\frac{\partial \Psi}{\partial \tilde{J}_{a}}\right) u_{a}^{\text {diss }}
\end{aligned}
$$

where we have used $\frac{d}{d t} l_{\tau, \sigma, \rho}=\dot{\theta}^{a} u_{a}^{\text {diss }}$, and since actuation is internal,

$$
C^{k}=\text { constant. }
$$

Assume that $E_{\tilde{\Phi}}$ has a local maximum at the equilibrium. Choose

$$
u_{a}^{\mathrm{diss}}=c_{a b}\left(\dot{\theta}^{b}+\frac{\partial \Psi}{\partial \tilde{J}_{b}}\right),
$$

where $c_{a b}$ is a positive definite matrix. Then,

$$
\frac{d}{d t} E_{\tilde{\Phi}}=c_{a b}\left(\dot{\theta}^{a}+\frac{\partial \Psi}{\partial \tilde{J}_{a}}\right)\left(\dot{\theta}^{b}+\frac{\partial \Psi}{\partial \tilde{J}_{b}}\right) \geq 0 .
$$

In the case that the equilibrium of interest is such that $\left.\dot{\theta}^{a}\right|_{e}=0$, we can take $\Psi$ as

$$
\Psi(\tilde{J})=\frac{1}{2} \epsilon^{b c} \tilde{J}_{b} \tilde{J}_{c},
$$

where $\epsilon^{b c}$ is a sign definite symmetric matrix. Then, (3.7) becomes

$$
\frac{d}{d t} E_{\tilde{\Phi}}=c_{a b}\left(\dot{\theta}^{a}+\epsilon^{a c} \tilde{J}_{c}\right)\left(\dot{\theta}^{b}+\epsilon^{b d} \tilde{J}_{d}\right) \geq 0 .
$$

To obtain asymptotic stability we use the LaSalle invariance principle and the details of the specific system, as illustrated below.

\section{GYROSCOPIC STABILIZATION WITH ROTORS}

We show how the preceding results on EulerPoincaré matching and stabilization apply to an important class of examples, namely rigid bodies carrying internal rotors. We can treat many systems, such as the spacecraft with internal rotors, the underwater vehicle with internal rotors and the heavy top with rotors, but we confine ourselves to the spacecraft here due to limited space.

Following Krishnaprasad [1985] and Bloch, Krishnaprasad, Marsden and Sánchez de Alvarez [1992], consider a rigid body with a rotor aligned along the third principal axis of the body as in Figure 4.1. The rotor spins under the influence of a torque $u$ acting on the rotor. The configuration space is $Q=S O(3) \times S^{1}$, with the first factor $H=S O(3)$ being the spacecraft attitude and the second factor $G=S^{1}$ being the rotor angle. The Lagrangian is total kinetic energy of the system, (rigid carrier plus rotor), with no potential energy.

The reduced Lagrangian on $\mathfrak{s o}(3) \times T S^{1}$ is

$$
l(\Omega, \dot{\phi})=\frac{1}{2}\left(\lambda_{1} \Omega_{1}^{2}+\lambda_{2} \Omega_{2}^{2}+I_{3} \Omega_{3}^{2}+J_{3}\left(\Omega_{3}+\dot{\phi}\right)^{2}\right)
$$

where $\Omega=\left(\Omega_{1}, \Omega_{2}, \Omega_{3}\right)$ is the body angular velocity vector of the carrier, $\phi$ is the relative angle of the rotor, $I_{1}>I_{2}>I_{3}$ are the rigid body moments of inertia, $J_{1}=J_{2}$ and $J_{3}$ are the rotor moments of inertia and $\lambda_{i}=I_{i}+J_{i}$. 


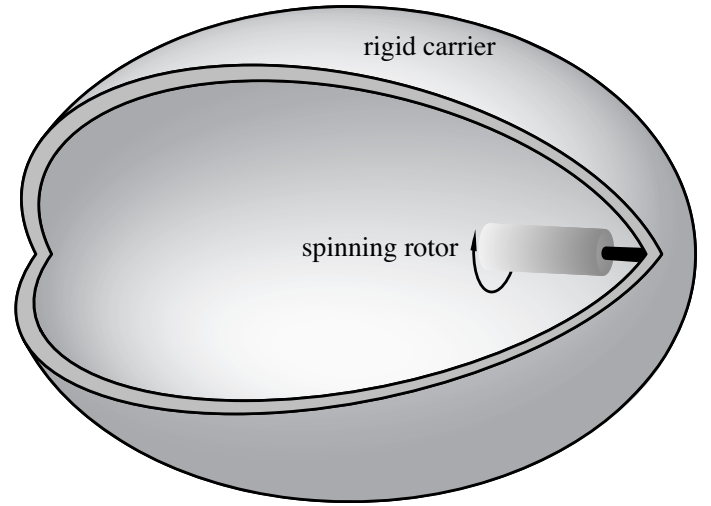

Fig. 4.1. The spacecraft with a rotor attached along the long axis.

The body angular momenta are determined by the Legendre transform to be

$$
\begin{aligned}
& \Pi_{1}=\tilde{M}_{1}=\lambda_{1} \Omega_{1}, \quad \Pi_{2}=\tilde{M}_{2}=\lambda_{2} \Omega_{2}, \\
& \Pi_{3}=\tilde{M}_{3}=\lambda_{3} \Omega_{3}+J_{3} \dot{\phi} .
\end{aligned}
$$

The momentum conjugate to $\phi$ is

$$
\frac{\partial l}{\partial \dot{\phi}}=l_{3}=J_{3}\left(\Omega_{3}+\dot{\phi}\right) \text {. }
$$

The equations of motion with a control torque $u$ acting on the rotor are

$$
\begin{aligned}
& \lambda_{1} \dot{\Omega}_{1}=\lambda_{2} \Omega_{2} \Omega_{3}-\left(\lambda_{3} \Omega_{3}+J_{3} \dot{\phi}\right) \Omega_{2} \\
& \lambda_{2} \dot{\Omega}_{2}=-\lambda_{1} \Omega_{1} \Omega_{3}+\left(\lambda_{3} \Omega_{3}+J_{3} \dot{\phi}\right) \Omega_{1} \\
& \lambda_{3} \dot{\Omega}_{3}+J_{3} \ddot{\phi}=\left(\lambda_{1}-\lambda_{2}\right) \Omega_{1} \Omega_{2} . \\
& i_{3}=u .
\end{aligned}
$$

Next, we form the controlled Lagrangian and apply the Euler-Poincaré matching theorem. Since the Abelian group $G=S^{1}$ is one-dimensional, $g_{a b}$, $\sigma_{a b}$ and $\rho_{a b}$ are all scalars. From (4.1), $g_{a b}=J_{3}$. Let $\sigma_{a b}=\sigma J_{3}$ and $\rho_{a b}=\rho J_{3}$ where $\sigma$ and $\rho$ are dimensionless scalars. For matching, choose $\tau_{\alpha}^{a}$ according to Assumption EP-1, i.e.,

$$
\left(\begin{array}{lll}
\tau_{\Omega_{1}}^{\phi} & \tau_{\Omega_{2}}^{\phi} & \tau_{\Omega_{3}}^{\phi}
\end{array}\right)=-\frac{1}{\sigma J_{3}}\left(\begin{array}{lll}
0 & 0 & J_{3}
\end{array}\right) .
$$

To satisfy Assumption EP-2, $\rho$ should satisfy

$$
\frac{1}{\sigma J_{3}}+\frac{1}{\rho J_{3}}=\frac{1}{J_{3}} \quad \text { i.e., } \quad \rho=\frac{\sigma}{\sigma-1} .
$$

Substituting into equation (2.5) with these choices, the controlled Lagrangian is given by

$$
\begin{aligned}
l_{\tau, \sigma, \rho} & =\frac{1}{2}\left(\lambda_{1} \Omega_{1}^{2}+\lambda_{2} \Omega_{2}^{2}+I_{3} \Omega_{3}^{2}+\frac{1}{\sigma} J_{3} \Omega_{3}^{2}\right) \\
& +\frac{1}{2}\left(\frac{\sigma}{\sigma-1} J_{3}\left(\Omega_{3}+\dot{\phi}-\frac{1}{\sigma} \Omega_{3}\right)^{2}\right)
\end{aligned}
$$

where $\sigma$ is a free variable and matching is ensured by Theorem 1 . The controlled conserved quantity is

$$
\tilde{l}_{3}=\tilde{J}=\frac{\partial l_{\tau, \sigma, \rho}}{\partial \dot{\phi}}=J_{3} \Omega_{3}+\rho J_{3} \dot{\phi}
$$

We use the formula (2.11) to get the control law

$$
u=u^{\mathrm{cons}}=k\left(\lambda_{1}-\lambda_{2}\right) \Omega_{1} \Omega_{2} .
$$

We note that

$$
\frac{1}{\sigma}=\frac{k}{1-k} \frac{I_{3}}{J_{3}}, \quad \frac{1}{\rho}=\frac{(1-k) J_{3}-k I_{3}}{(1-k) J_{3}}
$$

Consider $\left(\Omega_{1}, \Omega_{2}, \Omega_{3}, \dot{\phi}\right)=(0, \bar{\Omega}, 0,0), \bar{\Omega} \neq 0$, the equilibrium corresponding to steady rotation about the intermediate axis (unstable for the uncontrolled spacecraft). In contrast to earlier work, we carry out our analysis on the Lagrangian side and we do not restrict the stability analysis to the zero level set of the conserved momentum.

The Casimir for this problem is the total angular momentum of the body plus rotor system. We let

$$
\begin{aligned}
C= & \frac{1}{2}\left(\left(\lambda_{1} \Omega_{1}\right)^{2}+\left(\lambda_{2} \Omega_{2}\right)^{2}\right. \\
& \left.+\left(\left(I_{3}+\frac{J_{3}}{\sigma}\right) \Omega_{3}+\frac{\sigma-1}{\sigma} \tilde{l}_{3}\right)^{2}\right) .
\end{aligned}
$$

The Lyapunov function becomes

$$
\begin{aligned}
& E_{\tilde{\Phi}}=\frac{1}{2}\left(\lambda_{1} \Omega_{1}^{2}+\lambda_{2} \Omega_{2}^{2}+\left(I_{3}+\frac{J_{3}}{\sigma}\right) \Omega_{3}^{2}+\frac{1}{\rho J_{3}} \tilde{l}_{3}^{2}\right) \\
& \quad+\Phi(C)+\Psi\left(\tilde{l}_{3}\right) .
\end{aligned}
$$

To satisfy (2.26) of Theorem 2, we require

$$
\left.\Phi^{\prime}\right|_{e} \lambda_{2} \bar{\Omega}_{2}=-\bar{\Omega}_{2}, \quad \text { i.e., }\left.\quad \Phi^{\prime}\right|_{e}=-\frac{1}{\lambda_{2}} .
$$

We show that $N_{\alpha \beta}=G_{\alpha \beta}+G_{\alpha \gamma} \tilde{H}^{\gamma \delta} G_{\delta \beta}$ can be made definite. The matrix $G_{\alpha \beta}=g_{\alpha \beta}-g_{a \alpha} \rho^{a b} g_{b \beta}$ is diagonal: $\operatorname{diag}\left(\lambda_{1}, \lambda_{2}, I_{3}+J_{3} / \sigma\right)$. The matrix $\tilde{H}^{\alpha \beta}$ defined by (2.30) is computed to be

$$
\begin{aligned}
\tilde{H} & =\operatorname{diag}\left(\left.\Phi^{\prime}\right|_{e},\left.\Phi^{\prime}\right|_{e}+\left.\Phi^{\prime \prime}\right|_{e} \lambda_{2}^{2} \bar{\Omega}^{2},\left.\Phi^{\prime}\right|_{e}\right) \\
& =\operatorname{diag}\left(-\frac{1}{\lambda_{2}},-\frac{1}{\lambda_{2}}+\left.\Phi^{\prime \prime}\right|_{e} \lambda_{2}^{2} \bar{\Omega}^{2},-\frac{1}{\lambda_{2}}\right) .
\end{aligned}
$$

$N_{\alpha \beta}$ is computed to be diagonal with diagonal elements

$$
\left(\lambda_{1}-\frac{\lambda_{1}^{2}}{\lambda_{2}},\left.\Phi^{\prime \prime}\right|_{e} \lambda_{2}^{4} \bar{\Omega}^{2}, I_{3}+\frac{J_{3}}{\sigma}-\frac{1}{\lambda_{2}}\left(I_{3}+\frac{J_{3}}{\sigma}\right)^{2}\right) .
$$

Since the first diagonal element is negative, we choose $\left.\Phi^{\prime \prime}\right|_{e}$ to be negative also and require that

$$
I_{3}+\frac{J_{3}}{\sigma}-\frac{1}{\lambda_{2}}\left(I_{3}+\frac{J_{3}}{\sigma}\right)^{2}<0
$$

This condition holds if $k>1-I_{3} / \lambda_{2}$ and $E_{\tilde{\Phi}}$ has a local maximum at the equilibrium of interest. So, by Theorem 2 we have proved

Proposition 3. For $k>1-I_{3} / \lambda_{2}$, the equilibrium $(0, \bar{\Omega}, 0,0)$ is nonlinearly stable for the feedback controlled system. 


\section{ASYMPTOTIC STABILITY OF SPACECRAFT WITH A ROTOR}

We consider one approach to asymptotic stabilization; others are examined in a future publication. Dissipation is introduced so that $E_{\tilde{\Phi}}$ becomes a Lyapunov function for the closed-loop system with the complete control law which is computed below. Any given initial condition uniquely determines the equilibrium that can be asymptotically stabilized; the conservation of the Casimir function (4.10) gives the magnitude as $\left\|\lambda_{2} \bar{\Omega}\right\|^{2}=$ $\|\Pi\|^{2}=\|\Pi(0)\|^{2}$ and the stability implies that the sign of $\bar{\Omega}$ is the same as that of $\Omega_{2}(0)$ since the flow stays near $(0, \bar{\Omega}, 0,0)$.

In the expression for $E_{\tilde{\Phi}}, \Psi$ is given by

$$
\Psi\left(\tilde{l}_{3}\right)=\frac{1}{2 \epsilon J_{3}} \tilde{l}_{3}^{2}
$$

with $\epsilon<0$ and $|\epsilon| \ll 1$. By (3.6)

$$
u^{\mathrm{diss}}=c\left(\dot{\phi}+\frac{1}{\epsilon J_{3}} \tilde{l}_{3}\right)=c\left(\frac{1}{\epsilon} \Omega_{3}+\left(1+\frac{\rho}{\epsilon}\right) \dot{\phi}\right)
$$

with $c>0$, and the complete control law is

$$
u=k\left(\lambda_{1}-\lambda_{2}\right) \Omega_{1} \Omega_{2}+(1-k) \frac{1}{\rho} u^{\text {diss }},
$$

where $\rho$ is given by (4.9). Suppose that the flow $\left(\Omega_{1}(t), \Omega_{2}(t), \Omega_{3}(t), \dot{\phi}(t)\right)$ satisfies $\dot{E}_{\tilde{\Phi}}=0$, equivalently $u^{\text {diss }}=0$. Then, since $\dot{\tilde{l}}_{3}=u^{\text {diss }}, \tilde{l}_{3}$ is constant. This implies that

$$
\begin{aligned}
& \dot{\phi}(t)=\dot{\phi}(0)=\text { constant } \\
& \Omega_{3}(t)=\Omega_{3}(0)=\text { constant. }
\end{aligned}
$$

Substituting these into (4.4), we get

$$
\Omega_{1} \Omega_{2}=0 \text {. }
$$

Since $\Omega_{2}(t)$ stays near $\bar{\Omega} \neq 0$ by stability, (5.1) implies that

$$
\Omega_{1}(t)=0
$$

for all $t$. Substitution of this into (4.3) gives

$$
\Omega_{2}(t)=\Omega_{2}(0)=\text { constant. }
$$

Substitute these two into (4.2) and we get

$$
\left(\left(\lambda_{2}-\lambda_{3}\right) \Omega_{3}-J_{3} \dot{\phi}\right) \Omega_{2}(0)=0
$$

or

$$
\left(\lambda_{2}-\lambda_{3}\right) \Omega_{3}-J_{3} \dot{\phi}=0
$$

since $\Omega_{2}(0) \neq 0$ by stability. We also have $u^{\text {diss }}=$ 0 , which is given by

$$
\Omega_{3}+(\epsilon+\rho) \dot{\phi}=0 .
$$

All we require on $\epsilon$ is that it should be negative and satisfy some inequality to guarantee the (Lyapunov) stability. We can find $\epsilon$ satisfying

$$
\left(\lambda_{2}-\lambda_{3}\right)(\epsilon+\rho)+J_{3} \neq 0
$$

such that the two equations in (5.3) and (5.4) are independent. Then $\Omega_{3}=\dot{\phi}=0$. Thus, the only possible flow satisfying $u^{\text {diss }}=0$ is

$$
\Omega_{1}(t)=\Omega_{3}(t)=\dot{\phi}(t)=0, \quad \Omega_{2}(t)=\Omega_{2}(0) .
$$

This implies that

$$
\begin{aligned}
& \left|\lambda_{2} \Omega_{2}(0)\right|^{2}=\left|\lambda_{1} \Omega_{1}\right|^{2}+\left|\lambda_{2} \Omega_{2}\right|^{2}+\left|\lambda_{3} \Omega_{3}+J_{3} \dot{\phi}\right|^{2} \\
& =\left.|| \Pi\right|^{2}=\left|\lambda_{2} \bar{\Omega}\right|^{2}
\end{aligned}
$$

and so $\Omega_{2}(0)=\bar{\Omega}$ by stability. Thus, the only possible flow satisfying $u^{\text {diss }}=0$ is the equilibrium. By the LaSalle invariance principle, it is asymptotically stable.

\section{References.}

Bloch, A.M., D.E. Chang, N.E Leonard and J.E. Marsden [2000] Controlled Lagrangians and the stabilization of mechanical systems II; Potential shaping and tracking, in preparation.

Bloch, A.M., P.S. Krishnaprasad, J.E. Marsden and G. Sánchez de Alvarez [1992] Stabilization of rigid body dynamics by internal and external torques. Automatica 28, 745-756.

Bloch, A.M., N.E. Leonard and J.E. Marsden [1997] Stabilization of mechanical systems using controlled Lagrangians, Proc. CDC 36, 23562361.

Bloch, A.M., N.E. Leonard and J.E. Marsden [1998] Matching and stabilization by the method of controlled Lagrangians, Proc. CDC 37, 1446-1451.

Bloch, A.M., N.E. Leonard and J.E. Marsden [1999a] Stabilization of the pendulum on a rotor arm by the method of controlled Lagrangians, Proc. ICRA, IEEE, 500- 505.

Bloch, A.M., N.E. Leonard and J.E. Marsden [1999b] Potential shaping and the method of controlled Lagrangians Proc. CDC 38, 16531657.

Bloch, A.M., N.E. Leonard and J.E. Marsden [2000a] Controlled Lagrangians and the stabilization of mechanical systems I: The first matching theorem, IEEE Trans. Auto. Control, to appear.

Bloch, A.M., N.E. Leonard and J.E. Marsden [2000b] Controlled Lagrangians and the stabilization of Euler-Poincaré mechanical systems, Preprint.

Krishnaprasad, P.S. [1985] Lie-Poisson structures, dual-spin spacecraft and asymptotic stability, Nonl. Anal. Th. Meth. and Appl. 9,10111035.

Marsden, J.E. and T.S. Ratiu [1994] Symmetry and Mechanics. Texts in Applied Mathematics, 17, Springer-Verlag. Second Edition, 1999.

C.A. Woolsey and N.E. Leonard [1999], Underwater vehicle stabilization by internal rotors, Proc. ACC 3417-3421. 